\title{
STRUCTURAL STABILITY AND GROUP COHOMOLOGY
}

\author{
BY
}

PHILIP J. FLEMING

\begin{abstract}
We prove a version of the theorem of Stowe concerning the stability of stationary points of a differentiable group action which is valid on Hilbert manifolds. This result is then used to show that the vanishing of certain cohomology groups is sufficient to guarantee structural semistability for a differentiable action of a group of finite type on a closed smooth manifold. We then apply this to groups of diffeomorphisms of the circle.
\end{abstract}

\section{CHAPTER I. INTRODUCTION}

This paper is concerned with certain types of stability for differentiable actions of a finitely presented group on a differentiable manifold. The results are of two types. In Chapter II we prove a variation of D. Stowe's stability theorem [20] which is valid for Hilbert manifolds. This is used in Chapter III to obtain a group-cohomological criterion for a type of structural semistability. We then apply this to obtain new results about groups of diffeomorphisms of the circle.

The definition of structural stability for group actions. Let $M$ be a smooth closed Riemannian manifold and let $\operatorname{Diff}^{k}(M), k>0$, be the space of $C^{k}$-diffeomorphisms of $M$ with the Whitney $C^{k}$-topology. Let Diff ${ }^{0}(M)$ denote the space of homeomorphisms of $M$ with the metric topology. A $C^{k}$-action of a group $G$ on $M$ is a group homomorphism $\alpha: G \rightarrow \operatorname{Diff}^{k}(M)$ and the set of all $C^{k}$-actions of $G$ on $M$ will be denoted by $\operatorname{Act}^{k}(G, M)$. If $G$ is finitely generated we may topologize $\operatorname{Act}^{k}(G, M)$ by choosing a finite set of generators $K$ of $G$ and giving $\operatorname{Act}^{k}(G, M)$ the relative topology as a subspace of $\left\{f: K \rightarrow \operatorname{Diff}^{k}(M) \mid f\right.$ any function $\}$. It is clear that this topology is independent of the choice of the finite set of generators.

Definition. An action $\alpha$ in $\operatorname{Act}^{k}(G, M)$ is said to be structurally stable if there is a neighborhood $U$ of $\alpha$ in $\operatorname{Act}^{k}(G, M)$ such that for all actions $\beta$ in $U$ there is a homeomorphism $h$ of $M$ such that for all $g$ in $G$

$$
\alpha(g) \circ h=h \circ \beta(g) \text {. }
$$

History. The theory of structural stability for the general group action is still in its infancy. There are no general theorems, but only studies of special classes of group actions. The most striking result is the theorem, which was conjectured by Smale [18, 19] and proved by Robbin [14] and Robinson [15], that an action of the integers

Received by the editors July 21, 1981 and, in revised form, February 11, 1982.

1980 Mathematics Subject Classification. Primary 58F10; Secondary 54H15.

Key words and phrases. Group of finite type, group action, Hilbert manifold, stability of stationary points, structural stability, group cohomology. 
generated by a $C^{1}$-diffeomorphism of a closed manifold $M$ which satisfies Smale's Axiom A and the Strong Transversality Condition is structurally stable. It is still unknown whether these hypotheses are necessary for structural stability.

The compact Lie groups form a class of groups for which the theory of structural stability is completely understood. Palais [10] proved that a $C^{k}$-action, $k \geqslant 1$, of a compact Lie group on a closed manifold is always structurally stable. Furthermore, the conjugating map $h$ in equation (1) may be taken to be a $C^{k}$-diffeomorphism.

The most important work done on the structural stability of actions of discrete groups other than finite groups or the integers was done by Pugh and Shub [12, 13]. Roughly speaking, their results are that a differentiable group action which has a structurally stable diffeomorphism in its center is structurally stable. Also, Camacho [3] defined the notion of a Morse-Smale action and showed that Morse-Smale actions of certain groups on two-dimensional manifolds are structurally stable. Palis [11] has shown that among all smooth diffeomorphisms of a given manifold satisfying Axiom A and the Strong Transversality Condition the ones with discrete centralizers contain an open dense subset. Using this he then proves that certain "almost trivial" actions of a free abelian group are structurally stable.

In conclusion, the work of P. R. G. Sad [16] deserves to be mentioned. He has found a structurally stable action of the free abelian group on two generators on a product of spheres.

The Local Rigidity Theorem of A. Weil and the Stability Theorem of D. Stowe. In this section the relation between the two results in the title will be demonstrated and their relation to this dissertation indicated.

Let $G$ be a finitely generated group and $H$ a Lie group. Let $\operatorname{Hom}(G, H)$ denote the space of group homomorphisms from $G$ to $H$ with the topology it inherits as a subspace of $\{f: K \rightarrow H \mid f$ any function $\}$ where $K$ is a finite set of generators for $G$. We say that $\alpha$ in $\operatorname{Hom}(G, H)$ is locally rigid if there is a neighborhood $U$ of $\alpha$ so that $\beta$ in $U$ implies that there is an $h$ in $H$ such that $h \alpha(g)=\beta(g) h$ for every $g$ in $G$.

Given $\alpha$ in $\operatorname{Hom}(G, H)$, we may define a linear action of $G$ on $\dot{H}$, the Lie algebra of $H$, by $g \cdot X=\operatorname{Ad}(\alpha(g))(X)$ where Ad denotes the adjoint action of $H$ on $\dot{H}$. Letting $H^{1}(G, \dot{H})$ denote the first cohomology of $G$ with respect to this action we can now state the theorem [21].

Weil's Local Rigidity TheOREM. If $H^{1}(G, \dot{H})=0$ then $\alpha$ is locally rigid.

The idea behind the main theorem of Chapter III is to replace the Lie group $H$ by $\operatorname{Diff}^{k}(M)$ and thus get a structural stability theorem. There are two main difficulties with this approach: $\operatorname{Diff}^{k}(M)$ is not a differentiable group (left multiplication is not differentiable), and one cannot expect a differentiable conjugating map because of eigenvalue problems.

The recent work of D. Stowe which will be described below provides the idea for a way out of both of the above mentioned difficulties. Let $G$ be a finitely generated group and $M$ be a finite dimensional smooth manifold (possibly noncompact). Topologize Diff ${ }^{1}(M)$ by requiring that a net $\left\{f_{a}\right\}$ converges if and only if the corresponding net $\left\{D f_{a}\right\}$ of homeomorphisms of $T M$, the tangent bundle of $M$, 
converges uniformly on compact subsets of $M$. (Note: If $M$ is compact this is the same topology as described in the first section.) Let $\operatorname{Act}^{1}(G, M)$ be defined as in the first section with the same relative topology.

Given an action $\alpha$ in $\operatorname{Act}^{1}(G, M)$ and a point $p$ in $M$, we say that $p$ is a stationary point for $\alpha$ if $\alpha(g) p=p$ for all $g$ in $G$. A stationary point $p$ is stable if for every neighborhood $U$ of $P$ in $M$ there is a neighborhood $V$ of $\alpha$ in $\operatorname{Act}^{1}(G, M)$ such that for all $\beta$ in $V, \beta$ has a stationary point in $U$.

When $p$ is a stationary point for an action $\alpha$ we may define a linear action of $G$ on $T_{p} M$, the tangent space to $M$ at $p$, by $g \cdot X=D \alpha(g)_{p}(X)$. Letting $H^{1}\left(G, T_{p} M\right)$ denote the first cohomology of $G$ with respect to this action the theorem may now be stated [20].

Stowe's Stability Theorem. If $H^{1}\left(G, T_{p} M\right)=0$ then $p$ is stable.

The following proof that Weil's theorem is a corollary of Stowe's theorem suggests both analogues of Weil's theorem in the theory of structural stability and their proofs from analogues of Stowe's theorem on infinite dimensional manifolds of maps.

Proposition. Stowe's Stability Theorem implies Weil's Local Rigidity Theorem.

Proof. Given $\alpha$ and $\beta$ in $\operatorname{Hom}(G, H)$ define an action $[\alpha, \beta]$ on $H$ by

$$
[\alpha, \beta](g)(h)=\alpha(g) h \beta(g)^{-1} .
$$

Clearly $[\alpha, \alpha]$ has $e$, the identity element of $H$, as a stationary point and if $\beta$ is close to $\alpha$ in $\operatorname{Hom}(G, H)$ then $[\alpha, \beta]$ is close to $[\alpha, \alpha]$ in $\operatorname{Act}^{1}(G, H)$. Hence, by Stowe's theorem, if $H^{1}\left(G, T_{e} H\right)=0$ then $e$ is a stable stationary point of $[\alpha, \alpha]$. This means that for $\beta$ sufficiently close to $\alpha$ there is an element $h_{0}$ in $H$ such that

$$
h_{0}=[\alpha, \beta](g) h_{0}=\alpha(g) h_{0} \beta(g)^{-1}
$$

for all $g$ in $G$. Therefore, $h_{0} \beta(g)=\alpha(g) h_{0}$ for all $g$ in $G$. To complete the proof we observe that $H^{1}\left(G, T_{e} H\right)=H^{1}(G, \dot{H})$ which follows from the fact that $\dot{H}=T_{e} H$ and $D[\alpha, \alpha](g)_{e}=\operatorname{Ad}(\alpha(g))$.

I would like to thank J. Robbin for bringing Stowe's work to my attention and especially my advisor Tom Farrell for his help and encouragement.

\section{Chapter II. STOWE'S THEOREM ON HilberT MaNifoldS}

In this chapter we will state and prove a version of Stowe's Stability Theorem which is valid for actions of a finitely presented group on a smooth Hilbert manifold.

The space of group actions on a smooth Hilbert manifold. Let $M$ be a smooth Hilbert manifold with a given metric (see [8] for relevant definitions concerning infinite dimensional manifolds). We say that a homeomorphism of $M$ is a $C^{k}$-diffeomorphism if it and its inverse possess $k$ continuous Frechet derivatives. Let $\operatorname{Diff}^{k}(M)$, $k \geqslant 0$, denote the space of all $C^{k}$-diffeomorphisms of $M$ with the topology of uniform convergence of all $k$ derivatives on bounded subsets of $M$. More precisely, a 
net of $C^{k}$-diffeomorphisms converges if all derivatives up to and including order $k$ converge uniformly on every bounded subset of $M$.

The space $\operatorname{Act}^{k}(G, M)$ is then defined as in Chapter $I$ when $G$ is finitely generated. It is a subspace of $\left\{f: K \rightarrow \operatorname{Diff}^{k}(M) \mid f\right.$ is any function $\}$ where $K$ is a finite set of generators for $G$. The definitions of stationary point and stable stationary point of an action $\alpha$ in $\operatorname{Act}^{k}(G, M)$ are the same as in the case that $M$ is finite dimensional (see Chapter I).

The operator $D R$. Given $\alpha$ in $\operatorname{Act}^{1}(G, M)$, where

$$
G=\left\langle g_{1}, \ldots, g_{n} \mid r_{1}, \ldots, r_{m}\right\rangle
$$

is a finitely presented group, we may associate an operator $D R$ to a stationary point $p$ as follows:

Let $w$ be a word in the generators $g_{1}, \ldots, g_{n}$ of $G$ and let $\partial w / \partial g_{i}, 1 \leqslant i \leqslant n$, be the formal sum of integer multiples of elements of $G$ defined inductively by rules (a), (b) and (c).

(a) $\partial e / \partial g_{i}=0$ where $e$ is the empty word.

(b)

$$
\frac{\partial g_{j}}{\partial g_{i}}= \begin{cases}0 & \text { if } i \neq j \\ 1 & \text { if } i=j\end{cases}
$$

(c) $\partial u v / \partial g_{i}=\partial u / \partial g_{i}+u \partial v / \partial g_{i}$ for all words $u$ and $v$.

$\left(\partial w / \partial g_{i}\right.$ is called the Fox derivative of $w$ with respect to $g_{i}$.) If $\partial w / \partial g_{i}=\Sigma_{g \in G} n_{g} g$ and $v$ is in $T_{p} M$ define $\partial w / \partial g_{i} \cdot v=\sum n_{g} D \alpha(g)_{p}(v)$. Since only finitely many of the $n_{g}$ are nonzero, this makes $\partial w / \partial g_{i}$ into a bounded linear operator on $T_{p} M$. Now define $D w:\left(T_{p} M\right)^{n} \rightarrow T_{p} M$ by

$$
D w\left(v_{1}, \ldots, v_{n}\right)=\sum_{i=1}^{n} \frac{\partial w}{\partial g_{i}} \cdot v_{i} .
$$

Finally, the operator $D R:\left(T_{p} M\right)^{n} \rightarrow\left(T_{p} M\right)^{m}$ is defined by

$$
D R(\bar{v})=\left(D r_{1}(\bar{v}), \ldots, D r_{m}(\bar{v})\right) \text { where } \bar{v}=\left(v_{1}, \ldots, v_{n}\right)
$$

and, recall, $r_{1}, \ldots, r_{m}$ are the relations among the generators of $G$. It is clear that $D R$ is a bounded linear operator. The following elementary fact from combinatorial group theory will be useful later.

Proposition A. Let $f:\left\{g_{1}, \ldots, g_{n}\right\} \rightarrow T_{p} M$ be any function. If $\left(f\left(g_{1}\right), \ldots, f\left(g_{n}\right)\right) \in$ ker $D R$ then $f$ determines a well-defined crossed homomorphism by insisting that $f$ satisfy $f(g h)=f(g)+g f(h)$. Conversely, if $f: G \rightarrow T_{p} M$ is a crossed homomorphism then $\left(f\left(g_{1}\right), \ldots, f\left(g_{n}\right)\right) \in \operatorname{ker} D R$.

The main theorem and some auxiliary lemmas. For $C^{2}$-actions of the finitely presented group $G$ on the smooth Hilbert manifold $M$ with stationary point $p$ we have

THEOREM 2.1. If $H^{1}\left(G, T_{p} M\right)=0$ and the image of $D R$ is closed then $p$ is stable. 
The proof of this theorem requires the following:

LEMma 1. Let $A: E \rightarrow F$ be a bounded linear operator on the Banach spaces $E$ and $F$, $K$ a convex subset of $E$ and $f: E \rightarrow F a C^{1}$-map satisfying $\left\|D f_{x}-A\right\| \leqslant \varepsilon$ for all $x$ in $K$. Then $|f(a)-f(b)-A(a-b)| \leqslant \varepsilon|a-b|$ for all $a$ and $b$ in $K$.

Lemma 2. Let $f: E \rightarrow F$ be a $C^{1}$-map between the Banach spaces $E$ and $F$ such that $f(0)=0$. If ker $D f_{0}$ splits $E$ and $\operatorname{Im} D f_{0}$ is closed in $F$ then, for any closed complement $W$ of $\operatorname{ker} D f_{0}$ in $E$, the origin is an isolated zero of $f \mid W$, the restriction of $f$ to $W$.

LEMMA 3. Let $C^{2}(U, \mathbf{R})$ denote the Banach space of $C^{2}$-maps from a neighborhood $U$ of the origin in a Hilbert space to the real numbers, $\mathbf{R}$. If $f$ is in $C^{2}(U, \mathbf{R})$ and has the origin as a nondegenerate critical point then there is a neighborhood $N$ of $f$ in $C^{2}(U, \mathbf{R})$ such that for all $g$ in $N$ there is a critical point $x_{g}$ of $g$ such that $x_{g}$ approaches the origin as $g$ approaches $f$.

The proof of Theorem 1 in the case where $M$ is a Hilbert space. We first introduce some notation.

$M=\mathbf{H}$ and $\alpha$ is a $C^{2}$-action on $\mathbf{H}$.

$\rho(g)=D \alpha(g)_{p}$ where $g \in G$ and $p$ is a stationary point of the $C^{2}$-action $\alpha$.

$F: \mathbf{H} \rightarrow \mathbf{H}^{n}$ is the $C^{2}$-map defined by

$$
F(v)=\left(\alpha\left(g_{1}\right) v-v, \ldots, \alpha\left(g_{n}\right) v-v\right) .
$$

$V \equiv \operatorname{ker} D F_{0}=\bigcap_{i=1}^{n} \operatorname{ker}\left(\rho\left(g_{i}\right)-I\right)=\bigcap_{g \in G} \operatorname{ker}(\rho(g)-I)$.

$W=\{w \in \mathbf{H} \mid\langle v, w\rangle=0$ for all $v \in V\}$.

$h: \mathbf{H} \rightarrow \mathbf{R}$ is the $C^{2}$-functional defined by

$$
h(x)=\sum_{i=1}^{n}\left|\alpha\left(g_{i}\right) x-x\right|^{2} .
$$

Without loss of generality assume that $p$ is the origin of $\mathbf{H}$ and note that $T_{0} \mathbf{H} \cong \mathbf{H}$. Let $H^{1}(G, \mathbf{H})$ denote the first cohomology of $G$ with respect to the bounded linear action $\rho$, and observe that $H^{1}(G, \mathbf{H})=0$ is equivalent to $\operatorname{ker} D R=$ $\operatorname{Im} D F_{0}$.

The proof is by contradiction. Given a sequence of $C^{2}$-actions $\beta_{i}$ which converge to $\alpha$ but do not have stationary points converging to the origin, we construct a sequence of "approximate cocycles". This sequence of "cocycles" then yields a sequence of vectors $y_{i}$ in $\mathbf{H}$. We then differentiate certain functionals $h_{i}$ associated to $\beta_{i}$ in the direction $y_{i}$ to arrive at the desired contradiction.

Assume there is an open ball $B$ about the origin in $\mathbf{H}$ and $C^{2}$-actions $\beta_{1}, \beta_{2}, \ldots$ such that:

(a) $h(x) \geqslant 0$ for all $x \in W \cap \bar{B}$ except $x=0$;

(b) for each $1 \leqslant i \leqslant n, \beta_{j}\left(g_{i}\right) \rightarrow \alpha\left(g_{i}\right), D \beta_{j}\left(g_{i}\right) \rightarrow D \alpha\left(g_{i}\right)$ and $D^{2} \beta_{j}\left(g_{i}\right) \rightarrow D^{2} \alpha\left(g_{i}\right)$ uniformly on $B$;

(c) define $h_{j}(x)=\sum_{i=1}^{n}\left|\beta_{j}\left(g_{i}\right) x-x\right|^{2}$, then $h_{j} \mid W \cap B$ has a nonzero critical point $x_{j} \in W \cap B$ such that $x_{j}$ approaches zero as $j$ approaches infinity. 
The fact that $B$ may be chosen to make condition (a) hold follows from Lemma 2. Condition (c) relies on Lemma 3 and the fact that $D^{2} h(0) \mid W$ is nondegenerate since

$$
D^{2} h(0)(x, y)=2 \sum_{i=1}^{n}\left\langle\rho\left(g_{i}\right) x-x, \rho\left(g_{i}\right) y-y\right\rangle \text {. }
$$

Condition (b) implies that $\beta_{j}(g) x_{j}$ approaches zero as $j$ approaches infinity for each $g$ in $G$ since this is true for $g=e$ by Lemma 3 and the set of elements of $G$ for which this holds is closed under left multiplication by generators. Similarly, letting $\xi_{j}(g, h)$ denote the supremum of $\left\|D \beta_{j}(g)_{x}-\rho(g)\right\|$ along the segment from $x_{j}$ to $\beta_{j}(h) x_{j}$, we see that for fixed $g, h \in G, \xi_{j}(g, h)$ approaches zero as $j$ approaches infinity. Define functions $u_{j}: G \rightarrow \mathbf{H}$ by

$$
u_{j}(g)=h_{j}\left(x_{j}\right)^{-1 / 2}\left(\beta_{j}(g) x_{j}-x_{j}\right) .
$$

The following three claims show that as $j$ approaches infinity, $u_{j}$ behaves more and more like an honest cocycle.

Claim 1. If $h_{0}, \ldots, h_{p}$ are elements of $G$, with $h_{0}$ the identity element of $G$, then

$$
\left|u_{j}\left(h_{1} \cdots h_{p}\right)-\sum_{i=0}^{p-1} \rho\left(h_{0} \cdots h_{i}\right) u_{j}\left(h_{i+1}\right)\right| \leqslant \sum_{i=1}^{p-1} \xi_{j}\left(h_{1} \cdots h_{i}, h_{i+1}\right)\left|u_{j}\left(h_{i+1}\right)\right|
$$

where we take the right side of (2) to be zero if $p=1$.

Claim 2. For fixed $h \in G$, the sequence $u_{j}(h)$ is bounded.

Claim 3. $\lim _{j \rightarrow \infty}\left|D R\left(u_{j}\left(g_{1}\right), \ldots, u_{j}\left(g_{n}\right)\right)\right|=0$.

Proof of Claim 1. Using the fact that $\left\|D \beta_{j}(g)_{x}-\rho(g)\right\| \leqslant \xi_{j}(g, h)$ for $x$ on the line segment connecting $x_{j}$ to $\beta_{j}(h) x_{j}$ and Lemma 1 , we have

$$
\left|\beta_{j}(g) \beta_{j}(h) x_{j}-\beta_{j}(g) x_{j}-\rho(g)\left(\beta_{j}(h) x_{j}-x_{j}\right)\right| \leqslant \xi_{j}(g, h)\left|\beta_{j}(h) x_{j}-x_{j}\right| .
$$

Hence,

$$
\left|\left(\beta_{j}(g) \beta_{j}(h) x_{j}-\beta_{j}(g) x_{j}\right)\left(h_{j}\left(x_{j}\right)\right)^{-1 / 2}-\rho(g) u_{j}(h)\right| \leqslant \xi_{j}(g, h)\left|u_{j}(h)\right| .
$$

If $p=1,(2)$ is trivial and if $p=2$, note

$$
\begin{aligned}
u_{j}(g h)= & \left(\beta_{j}(g h) x_{j}-x_{j}\right)\left(h_{j}\left(x_{j}\right)\right)^{-1 / 2} \\
= & \left(\beta_{j}(g) \beta_{j}(h) x_{j}-\beta_{j}(g) x_{j}\right)\left(h_{j}\left(x_{j}\right)\right)^{-1 / 2} \\
& +\left(\beta_{j}(g) x_{j}-x_{j}\right)\left(h_{j}\left(x_{j}\right)\right)^{-1 / 2} .
\end{aligned}
$$

So,

$$
\left|u_{j}(g)+\rho(g) u_{j}(h)-u_{j}(g h)\right| \leqslant \xi_{j}(g, h)\left|u_{j}(h)\right| .
$$

We now proceed by induction and assume (2) is true for $p=q$. Adding

$$
\begin{aligned}
\mid u_{j}\left(h_{1} \cdots h_{q}\right) & +\rho\left(h_{1} \cdots h_{q}\right) u_{j}\left(h_{q+1}\right)-u_{j}\left(h_{1} \cdots h_{q+1}\right) \mid \\
& \leqslant \xi_{j}\left(h_{1} \cdots h_{q}, h_{q+1}\right)\left|u_{j}\left(h_{q+1}\right)\right|
\end{aligned}
$$

to (2) and using the triangle inequality, we get (2) for $p=q+1$. 
Proof of Claim 2. By Claim 1 it suffices to establish this claim when $h$ is either a generator, the inverse of a generator, or the identity $e$ of $G$. It is easy to see that $u_{j}(e)=0$ for all $j$ and

$$
\begin{aligned}
\left|u_{j}\left(g_{i}\right)\right| & =\left|\beta_{j}\left(g_{i}\right) x_{j}-x_{j}\right|\left(\sum_{p=1}^{n}\left|\beta_{j}\left(g_{p}\right) x_{j}-x_{j}\right|^{2}\right)^{-1 / 2} \\
& \leqslant 1 \text { for all } j \text { and all } 1 \leqslant i \leqslant n .
\end{aligned}
$$

Also, using (2), we see that

$$
\left|u_{j}\left(g_{i}^{-1}\right)+\rho\left(g_{i}^{-1}\right) u_{j}\left(g_{i}\right)-u_{j}\left(g_{i} g_{i}^{-1}\right)\right| \leqslant \xi_{j}\left(g_{i}, g_{i}^{-1}\right)\left|u_{j}\left(g_{i}\right)\right|
$$

Hence,

$$
\begin{aligned}
\left|u_{j}\left(g_{i}^{-1}\right)\right| & \leqslant \xi_{j}\left(g_{i}, g_{i}^{-1}\right)\left|u_{j}\left(g_{i}\right)\right|+\left\|\rho\left(g_{i}^{-1}\right)\right\|\left|u_{j}\left(g_{i}\right)\right| \\
& \leqslant \xi_{j}\left(g_{i}, g_{i}^{-1}\right)+\left\|\rho\left(g_{i}^{-1}\right)\right\| .
\end{aligned}
$$

Proof of Claim 3. Let $r_{s}=h_{1} \cdots h_{p}$ be a relator where $h_{k}$ is either a generator or the inverse of a generator and observe

$$
\frac{\partial r_{s}}{\partial g_{i}}=\sum_{k=0}^{p-1} h_{0} \cdots h_{k} \frac{\partial h_{k+1}}{\partial g_{i}} \quad \text { where } h_{0}=e
$$

Also,

$$
\left|\sum_{i=1}^{n} \rho\left(\frac{\partial h_{k+1}}{\partial g_{i}}\right) u_{j}\left(g_{i}\right)-u_{j}\left(h_{k+1}\right)\right|=0
$$

if $h_{k+1}$ is a generator. And for $h_{k+1}=g_{q}^{-1}$, for some $1 \leqslant q \leqslant n$, we have

$$
\left|\sum_{i=1}^{n} \rho\left(\frac{\partial h_{k+1}}{\partial g_{i}}\right) u_{j}\left(g_{i}\right)-u_{j}\left(h_{k+1}\right)\right|=\left|u_{j}\left(g_{q}^{-1}\right)+\rho\left(g_{q}^{-1}\right) u_{j}\left(g_{q}\right)\right|,
$$

which approaches zero as $j$ approaches infinity. Finally, note that

$$
\begin{aligned}
\lim _{j \rightarrow \infty}\left|\sum_{k=0}^{p-1} \rho\left(h_{0} \cdots h_{k}\right) u_{j}\left(h_{k+1}\right)\right| \\
\quad=\lim _{j \rightarrow \infty}\left|u_{j}\left(h_{0} \cdots h_{p}\right)-\sum_{k=0}^{p-1} \rho\left(h_{0} \cdots h_{k}\right) u_{j}\left(h_{k+1}\right)\right| \\
\quad \leqslant \lim _{j \rightarrow \infty} \sum_{k=1}^{p-1} \xi_{j}\left(h_{1} \cdots h_{k}, h_{k+1}\right)\left|u_{j}\left(h_{k+1}\right)\right| \\
=0 \text { by Claim 2. }
\end{aligned}
$$


The following inequality finishes the proof.

$$
\begin{aligned}
\left|\operatorname{Dr}_{s}\left(u_{j}\left(g_{1}\right), \ldots, u_{j}\left(g_{n}\right)\right)-\sum_{k=0}^{p-1} \rho\left(h_{0} \cdots h_{k}\right) u_{j}\left(h_{k+1}\right)\right| \\
\quad=\left|\sum_{i=1}^{n} \sum_{k=0}^{p-1} \rho\left(h_{0} \cdots h_{k} \cdot \frac{\partial h_{k+1}}{\partial g_{i}}\right) u_{j}\left(g_{i}\right)-\sum_{k=0}^{p-1} \rho\left(h_{0} \cdots h_{k}\right) u_{j}\left(h_{k+1}\right)\right| \\
\quad \leqslant \sum_{k=0}^{p-1}\left\|\rho\left(h_{0} \cdots h_{k}\right)\right\|\left|\sum_{i=1}^{n} \rho\left(\frac{\partial h_{k+1}}{\partial g_{i}}\right) u_{j}\left(g_{i}\right)-u_{j}\left(h_{k+1}\right)\right| .
\end{aligned}
$$

Hence, $\operatorname{Dr}_{s}\left(u_{j}\left(g_{1}\right), \ldots, u_{j}\left(g_{n}\right)\right)$ approaches zero as $j$ approaches infinity for all $1 \leqslant s \leqslant m$. This complete the proof of Claim 3 .

We now construct a bounded sequence $y_{j}$ in $W$ such that

$$
\lim _{j \rightarrow \infty} D_{y_{j}} \tilde{h}_{j}\left(x_{j}\right)\left(h_{j}\left(x_{j}\right)\right)^{-1 / 2}=-2,
$$

where $\tilde{h}_{j}=h_{j} \mid W \cap B$ and $D_{y_{j}}$ denotes the directional derivative. This contradicts the fact that $x_{j}$ is a critical point of $\tilde{h}_{j}$.

Let $\bar{u}_{j}=\left(u_{j}\left(g_{1}\right), \ldots, u_{j}\left(g_{n}\right)\right)=\bar{v}_{j}+\bar{w}_{j}$, where $\bar{v}_{j} \in \operatorname{ker} D R$ and $\bar{w}_{j} \in(\operatorname{ker} D R)^{\perp}$. By Claim $3, \lim _{j \rightarrow \infty}\left|D R\left(\bar{w}_{j}\right)\right|=0$. Since $D R$, restricted to $(\operatorname{ker} D R)^{\perp}$, is injective and has closed image, it satisfies $|D R(x)| \geqslant \delta|x|$ for some constant $\delta>0$ (see [4]). This implies that $\lim _{j \rightarrow \infty} \bar{w}_{j}=0$. Hence,

$$
\lim _{j \rightarrow \infty}\left|\bar{u}_{j}-\bar{v}_{j}\right|=0 \text {. }
$$

By Proposition A and the hypothesis that $H^{1}(G, \mathbf{H})=0$, we have

$$
\bar{v}_{j}=\left(y_{j}-\rho\left(g_{1}\right) y_{j}, \ldots, y_{j}-\rho\left(g_{n}\right) y_{j}\right)
$$

for some $y_{j} \in \mathbf{H}$. If we choose $y_{j} \in W$ then, since $D F_{0}$ restricted to $W$ is injective and has closed image (recall, $\operatorname{Im} D F_{0}=\operatorname{ker} D R$ ), we have $\delta\left|y_{j}\right| \leqslant\left|D F_{0}\left(y_{j}\right)\right|=\left|\bar{v}_{j}\right|$. Hence $y_{j}$ is a bounded sequence because $\bar{u}_{j}$ is a bounded sequence. Now note that

$$
D_{y_{j}} \tilde{h}_{j}\left(x_{j}\right)=2 \sum_{i=1}^{n}\left\langle\beta_{j}\left(g_{i}\right) x_{j}-x_{j}, D \beta_{j}\left(g_{i}\right)_{x_{j}} y_{j}-y_{j}\right\rangle \text {. }
$$

Hence,

$$
D_{y_{j}} \tilde{h}_{j}\left(x_{j}\right)\left(h_{j}\left(x_{j}\right)\right)^{-1 / 2}=2 \sum_{i=1}^{n}\left\langle u_{j}\left(g_{i}\right), D \beta_{j}\left(g_{i}\right)_{x_{j}} y_{j}-y_{j}\right\rangle
$$

And so

$$
\begin{aligned}
\mid D_{y_{i}} \tilde{h}_{j}\left(x_{j}\right)( & \left.h_{j}\left(x_{j}\right)\right)^{-1 / 2}+2 \mid \\
& =\left|D_{y_{j}} \tilde{h}_{j}\left(x_{j}\right)\left(h_{j}\left(x_{j}\right)\right)^{-1 / 2}+2 \sum_{i=1}^{n}\left\langle u_{j}\left(g_{i}\right), u_{j}\left(g_{i}\right)\right\rangle\right| \\
& =\left|2 \sum_{i=1}^{n}\left\langle u_{j}\left(g_{i}\right), D \beta_{j}\left(g_{i}\right)_{x_{j}} y_{j}-y_{j}+u_{j}\left(g_{j}\right)\right\rangle\right| .
\end{aligned}
$$


Claim 4. $\lim _{j \rightarrow \infty}\left|D \beta_{j}\left(g_{i}\right)_{x_{j}} y_{j}-y_{j}+u_{j}\left(g_{i}\right)\right|=0$ for $1 \leqslant i \leqslant n$.

Proof of Claim 4. We have

$$
\lim _{j \rightarrow \infty}\left|D \beta_{j}\left(g_{i}\right)_{x_{j}} y_{j}-y_{j}-\left(\rho\left(g_{i}\right) y_{j}-y_{j}\right)\right| \leqslant \lim _{j \rightarrow \infty}\left\|D \beta_{j}\left(g_{i}\right)_{x_{j}}-\rho\left(g_{i}\right)\right\|\left|y_{i}\right|=0
$$

since $y_{j}$ is bounded and $D \beta_{j}\left(g_{i}\right)$ converges uniformly to $D \alpha\left(g_{i}\right)$ on $B$. Equations (3) and (4) now yield the claim. The claim implies that

$$
\lim _{j \rightarrow \infty} D_{y_{j}} \tilde{h}_{j}\left(x_{j}\right)\left(h_{j}\left(x_{j}\right)\right)^{-1 / 2}=-2 \text {, }
$$

contradicting the fact that $x_{j}$ is a critical point of $h_{j}$. This completes the proof in the case where $M$ is a Hilbert space.

The proof in the general case. We now show that with suitable care the argument given in the last section may be made in coordinate charts about the stationary point $p$.

Let $U^{\prime}$ be an open neighborhood of the origin of a Hilbert space $\mathbf{H}$ and $\psi: U \rightarrow U^{\prime}$ a coordinate chart at $p$ such that $\psi(p)=0$. Define a bounded linear action $\rho$ of $G$ on $\mathbf{H}$ as follows. Let $U_{g}^{\prime}=\psi\left(U \cap \alpha(g)^{-1}(U)\right)$ and $S=\left\{(g, x) \in G \times \mathbf{H} \mid x \in U_{g}^{\prime}\right\}$. It is easy to see that $S$ is open in $G \times \mathbf{H}, G \times\{0\}$ is contained in $S$, and $\alpha^{\prime}(g)=\psi \circ \alpha(g) \circ \psi^{-1}: U_{g}^{\prime} \rightarrow U^{\prime}$ is a $C^{2}$-embedding. Since $0 \in U_{g}^{\prime}$ for all $g \in G$, we may define $\rho$ by $\rho(g)=D \alpha^{\prime}(g)_{0}$. Clearly, $H^{1}\left(G, T_{p} M\right) \approx H^{1}(G, \mathbf{H})$ and $D R$ has closed image if and only if $D R^{\prime}$ has closed image, where $D R^{\prime}$ is the operator associated to $\rho$. In the proof we show that if $H^{1}(G, \mathbf{H})=0$ and $D R^{\prime}$ has closed image then $p$ is stable.

Let $V=\bigcap_{i=1}^{n} \operatorname{ker}\left(\rho\left(g_{i}\right)-I\right)$ and $W=V^{\perp}$. Define $h: \bigcap_{i=1}^{n} U_{g_{i}}^{\prime} \rightarrow \mathbf{R}$ by

$$
h(x)=\sum_{i=1}^{n}\left|\alpha^{\prime}\left(g_{i}\right) x-x\right|^{2} .
$$

The proof, as in the last section, is by contradiction. Assume that there is an open set $B$ about $p$ in $M$ and $C^{2}$-actions $\beta_{1}, \beta_{2}, \ldots$ converging to $\alpha$ such that

(1) $\overline{B^{\prime}} \equiv \psi(\bar{B}) \subseteq \cap_{i=1}^{n} U_{g_{j}}^{\prime}$,

(2) $\beta_{j}\left(g_{i}\right)(\bar{B}) \subset U$ for all $j \geqslant 1$ and $1 \leqslant i \leqslant n$.

Condition (2) implies that all of the $\beta_{j}$ are close enough to $\alpha$ so that $\beta_{j}^{\prime}\left(g_{i}\right)=$ $\psi \circ \beta_{j}\left(g_{i}\right) \circ \psi^{-1}$ is defined on $\bar{B}^{\prime}$ for $j \geqslant 1$ and $1 \leqslant i \leqslant n$.

Claim 1. For every $g \in G$ there is a ball $B_{g}^{\prime}$ about $0 \in \mathbf{H}$ and an integer $I_{g}$ such that $\beta_{i}^{\prime}(g)$ is defined on $B_{g}^{\prime}$ for $i \geqslant I_{g}$.

Proof. It suffices to show that the subset of $G$ for which the claim is true is nonempty and closed under right multiplication by generators of $G$. To see that it is nonempty, take $B_{e}^{\prime}=B^{\prime}$ and $I_{e}=1$ where $e$ is the identity element of $G$. Now, if $\beta_{j}^{\prime}(g)$ is defined on $B_{g}^{\prime}$ for $i \geqslant I_{g}$ then $\beta_{j}^{\prime}\left(g g_{i}\right)=\beta_{j}^{\prime}(g) \circ \beta_{j}^{\prime}\left(g_{i}\right)$ is defined on $B_{g}^{\prime} \cap \beta_{j}^{\prime}\left(g_{i}\right)^{-1}\left(B^{\prime}\right)$, which is nonempty and contains the origin for sufficiently large $j$ because $\beta_{j}^{\prime}\left(g_{i}\right) \rightarrow \alpha^{\prime}\left(g_{i}\right)$ uniformly on $B^{\prime}$. This completes the proof of the claim.

Now define $h_{j}: B^{\prime} \rightarrow \mathbf{R}$ by

$$
h_{j}(x)=\sum_{i=1}^{n}\left|\beta_{j}^{\prime}\left(g_{i}\right) x-x\right|^{2} .
$$


By Lemma 3 we may assume that $h_{j} \mid W \cap \bar{B}^{\prime}$ has a nonzero critical point $x_{j} \in B^{\prime}$, $j \geqslant 1$. By Lemma 2 the origin is an isolated nondegenerate critical point of $h \mid W \cap \bar{B}^{\prime}$. Using Claim 1, for every $g \in G$ there is an integer $N_{g}$ such that $\beta_{j}^{\prime}(g) x_{j}$ is defined (i.e. $x_{j} \in B_{j}^{\prime}$ ) for $j \geqslant N_{g}$. It is then easy to see that $\beta_{j}^{\prime}(g) x_{j}$ approaches zero as $j$ approaches infinity by an argument similar to the proof of Claim 1.

Given $g, h \in G$, it is now easy to see that the line segment joining $x_{j}$ and $\beta_{j}^{\prime}(h) x_{j}$ is contained in $B_{g}^{\prime}$ for sufficiently large $j$ and that $\xi_{j}(g, h)$, the supremum of $\left\|D \beta_{j}^{\prime}(g)_{x}-\rho(g)\right\|$ along this segment, tends to zero as $j$ tends to infinity. Now define

$$
u_{j}(g)=\left(\beta_{j}^{\prime}(g) x_{j}-x_{j}\right)\left(h_{j}\left(x_{j}\right)\right)^{-1 / 2}
$$

for all $j \geqslant N_{g}$.

Claim 2. There is an integer $M\left(h_{1}, \ldots, h_{k}\right)$ for any elements $h_{1}, \ldots, h_{k} \in G, k \geqslant 2$, such that for $j \geqslant M$,

$$
\left|\sum_{i=0}^{k-1} \rho\left(h_{0} \cdots h_{i}\right) u_{j}\left(h_{i+1}\right)-u_{j}\left(h_{1} \cdots h_{k}\right)\right| \leqslant \sum_{i=1}^{k-1} \xi_{j}\left(h_{1} \cdots h_{i}, h_{i+1}\right)\left|u_{j}\left(h_{j+1}\right)\right|
$$

where $h_{0}$ is the identity element of $G$.

The proof of this claim is the same as the proof of Claim 1 of the last section once $u_{j}$ is defined on the appropriate elements of $G$.

Claim 3. For fixed $g \in G$, the sequence $u_{j}(g)$ is bounded. Again the proof of this claim is identical to the proof of Claim 2 of the last section.

Claim 4. $\lim _{j \rightarrow \infty}\left|D R^{\prime}\left(u_{j}\left(g_{1}\right), \ldots, u_{j}\left(g_{n}\right)\right)\right|=0$.

This is Claim 3 of the last section. The remainder of the proof in the general case is identical to the case $M=\mathbf{H}$ given in the last section if we replace $\alpha$ by $\alpha^{\prime}$ and $\beta_{j}$ by $\beta_{j}^{\prime}$.

More on $D R$. Let $G=\left\langle g_{1}, \ldots, g_{n} \mid r_{1}, \ldots, r_{m}\right\rangle$ be a finitely presented group. Following [2] we say that $G$ is of type $(F P)_{n}, n \geqslant 0$, if there is a projective resolution of the trivial $G$-module $\mathbf{Z}$

$$
\rightarrow P_{2} \rightarrow P_{1} \rightarrow P_{0} \rightarrow \mathrm{Z} \rightarrow 0
$$

such that $P_{i}$ is finitely generated over $\mathbf{Z} G$ for $0 \leqslant i \leqslant n$. Here, $\mathbf{Z}$ denotes the integers and $\mathbf{Z} G$ denotes the so-called group-ring of $G$ consisting of all finite formal sums of integer multiples of elements of $G$.

Proposition 2.2. Let $\mathbf{H}$ be a Hilbert space on which $G$ acts by bounded linear operators. If $G$ is of type $(F P)_{3}$ and $H^{2}(G, \mathbf{H})$ is finite dimensional then the image of $D R$ is closed.

The following three lemmas are needed in the proof of Proposition 2.2. If $M$ is a $G$-module generated by $a_{1}, \ldots, a_{p}$ and $\mathbf{H}$ is a $G$-Hilbert space (that is, a Hilbert space on which $G$ acts by bounded linear operators) then $\operatorname{Hom}_{G}(M, \mathbf{H})$ is a Hilbert space with norm given by

$$
|f|^{2}=\sum_{i=1}^{p}\left|f\left(a_{i}\right)\right|^{2}
$$

In the following we suppress the subscript $G$ in $\operatorname{Hom}_{G}$. 
Lemma A. If $\psi: M \rightarrow N$ is a G-homomorphism between finitely generated $G$-modules then $\psi^{*}: \operatorname{Hom}(N, \mathbf{H}) \rightarrow \operatorname{Hom}(M, \mathbf{H})$ is continuous with respect to the norm topology.

Proof. First note that the topology induced by the norm is independent of the choice of generators. Let $a_{1}, \ldots, a_{p}$ generate $M$ and let $b_{1}, \ldots, b_{q}$ generate $N$. Let $\psi\left(a_{i}\right)=\sum_{j=1}^{q} \gamma_{j}^{i} b_{j}$ where $\gamma_{j}^{i} \in \mathbf{Z} G, 1 \leqslant i \leqslant p, 1 \leqslant j \leqslant q$. We have

$$
\begin{aligned}
\left|\psi^{*} f\right|^{2} & =\sum_{i=1}^{p}\left|f\left(\psi\left(a_{i}\right)\right)\right|^{2}=\sum_{i=1}^{p}\left|f\left(\sum_{j=1}^{q} \gamma_{j}^{i} b_{j}\right)\right|^{2} \\
& =\sum_{i=1}^{p}\left|\sum_{j=1}^{q} \gamma_{j}^{i} f\left(b_{j}\right)\right|^{2} \leqslant \sum_{i=1}^{p} \sum_{j=1}^{q}\left\|\gamma_{j}^{i}\right\|^{2}\left|f\left(b_{j}\right)\right|^{2} \\
& \leqslant C|f|^{2}
\end{aligned}
$$

where $C=\max _{1 \leqslant j \leqslant q} \sum_{i=1}^{p}\left\|\gamma_{j}^{i}\right\|^{2}$ and \|\| denotes the operator norm.

This shows that $\psi^{*}$ is a bounded linear operator and hence is continuous.

Let $F$ be a finitely generated free group that maps onto $G$ and let $R$ denote the kernel of this surjection. Let $R_{\mathrm{ab}}=R /[R, R]$ where $[R, R]$ denotes the commutator subgroup of $R$. The abelian group $R_{\mathrm{ab}}$ has a natural $G$-module structure and if $G$ is finitely presented, $R_{\mathrm{ab}}$ is finitely generated as a $\mathbf{Z} G$-module (see [2]). Let $\pi$ : $F R \rightarrow R_{\mathrm{ab}}$ be an epimorphism of the finitely generated free $\mathrm{Z} G$-module $F R$ to $R_{\mathrm{ab}}$ and let $K=\operatorname{ker} \pi$.

LEMMA B. If $G$ is of type $(F P)_{3}$ then $K$ is finitely generated over $\mathbf{Z} G$.

Proof. Consider the resolution of $\mathbf{Z}$,

$$
0 \rightarrow K \stackrel{j}{\rightarrow} F R \stackrel{\partial \circ \pi}{\rightarrow} E G \stackrel{\theta}{\rightarrow} \mathbf{Z} G \stackrel{\varepsilon}{\rightarrow} \mathbf{Z} \rightarrow 0,
$$

where $\varepsilon\left(\Sigma n_{g} g\right)=\Sigma n_{g}, E G$ is the free $\mathbf{Z} G$-module on the symbols $e_{1}, \ldots, e_{n}, \theta\left(e_{i}\right)=$ $g_{i}-1, \partial: R_{\mathrm{ab}} \rightarrow E G$ is the map $\partial w=\sum_{i=1}^{n} \partial w e_{i} / \partial g_{i}$ and $j$ is inclusion (see [17]). On the other hand, by hypothesis we have a projective resolution

$$
\rightarrow P_{3} \rightarrow P_{2} \rightarrow P_{1} \rightarrow P_{0} \rightarrow \mathrm{Z} \rightarrow 0
$$

where $P_{0}, P_{1}, P_{2}$, and $P_{3}$ are finitely generated. Letting $L=\operatorname{ker}\left(P_{2} \rightarrow P_{1}\right)$ we have the resolution

$$
0 \rightarrow L \rightarrow P_{2} \rightarrow P_{1} \rightarrow P_{0} \rightarrow \mathbf{Z} \rightarrow 0 .
$$

We now apply the generalized Schanuel lemma (see [7]) and conclude

$$
K \oplus P_{2} \oplus E G \oplus \mathbf{Z} G \cong L \oplus F R \oplus P_{1} \oplus P_{0} .
$$

Observing that $L$ is the image of the finitely generated module $P_{3}$ and hence is finitely generated completes the proof of Lemma B.

$$
\text { Lemma C. } D R=\partial^{*}: \operatorname{Hom}(E G, \mathbf{H}) \rightarrow \operatorname{Hom}\left(R_{\mathrm{ab}}, \mathbf{H}\right) \text {. }
$$


Proof. It is easy to see that every element of $R_{\mathrm{ab}}$ is of the form $\sum_{i=1}^{m} \gamma_{i} r_{i}$ where $r_{i}$ is a relation of $G$ and $\gamma_{i} \in \mathbf{Z} G$. Now calculate,

$$
\begin{aligned}
\partial^{*}(f)\left(\sum_{i=1}^{m} \gamma_{i} r_{i}\right) & =\sum_{i=1}^{m} \gamma_{i} f\left(\partial r_{i}\right)=\sum_{i=1}^{m} \gamma_{i} f\left(\sum_{j=1}^{n} \frac{\partial r_{i}}{\partial g_{j}} e_{j}\right) \\
& =\sum_{i=1}^{m} \gamma_{i} D r_{i}\left(f\left(e_{1}\right), \ldots, f\left(e_{n}\right)\right) .
\end{aligned}
$$

Proof of Proposition 2.2. We have the following exact sequence of finitely generated $G$-modules:

$$
\begin{aligned}
0 \rightarrow K \stackrel{j}{\rightarrow} & F R \stackrel{\partial \stackrel{\pi}{\rightarrow}}{\rightarrow} E G \stackrel{\theta}{\rightarrow} \mathbf{Z} G \stackrel{\varepsilon}{\rightarrow} \mathbf{Z} \rightarrow 0 \\
\pi \downarrow & \nearrow \partial \\
R_{\mathrm{ab}} &
\end{aligned}
$$

Applying $\operatorname{Hom}(-, \mathbf{H})$ we get

$$
\begin{aligned}
\operatorname{Hom}(K, \mathbf{H}) \stackrel{j^{*}}{\leftarrow} \operatorname{Hom}(F R, \mathbf{H}) \leftarrow & \\
& \stackrel{\pi^{*} \circ \partial^{*}}{\leftarrow} \operatorname{Hom}(E G, \mathbf{H}) \stackrel{\theta^{*}}{\leftarrow} \operatorname{Hom}(\mathbf{Z} G, \mathbf{H})
\end{aligned}
$$

where all maps are continuous by Lemma A. Since $H^{2}(G, \mathbf{H})$ is finite dimensional by hypothesis we see that the image of $\pi^{*} \circ \partial^{*}$ has finite codimension in ker $j^{*}$ and hence is closed. That means $\pi^{*}\left(\operatorname{Im} \partial^{*}\right)$ is closed and so $\pi^{*-1}\left(\pi^{*}\left(\operatorname{Im} \partial^{*}\right)\right)$ is closed. Since $\pi^{*}$ is injective this implies that $\operatorname{Im} \partial^{*}$ is closed. Lemma C now completes the proof.

COROllaRy 2.3. If $G$ is a finite group acting on a Hilbert space by bounded linear operators then DR has closed image.

Proof. It is easily seen that a finite group is $(F P)_{n}$ for all $n$ and that $H^{2}(G, \mathbf{H})$ is zero regardless of the particular action.

The next proposition says that the set of actions for which $D R$ has closed image is closed under taking free products.

Let $\alpha$ and $\beta$ be continous linear actions of the finitely presented groups $G$ and $H$, respectively, on the topological vector space $V$. Define an action $\alpha * \beta$ of the free product of $G$ and $H, G * H$ on $V$ by $\alpha * \beta(g)=\alpha(g)$ if $g \in G$ and $\alpha * \beta(h)=\beta(h)$ if $h \in H$, and by requiring that $\alpha * \beta$ actually be an action.

Proposition 2.4. If the operators associated to $\alpha$ and $\beta$ both have closed images then the operator associated to $\alpha * \beta$ has closed image.

The proof of this proposition is immediate. We close this chapter with the following theorem.

THEOREM 2.5. If $G$ is a free product of finite groups and free groups then $D R$ is always closed.

Proof. This is a consequence of Corollary 2.3 and Proposition 2.4. 


\section{Chapter III. $S$-STRUCTURAL STABILITY}

In this chapter we prove an analogue of Weil's Local Rigidity Theorem for differentiable actions of finitely presented groups. We then apply this result to actions on the circle.

A stability theorem. Let $G=\left\langle g_{1}, \ldots, g_{n} \mid r_{1}, \ldots, r_{m}\right\rangle$ be a finitely presented group and let $M^{d}$ be a smooth closed Riemannian manifold of dimension $d$. Given a $C^{k}$-action $\alpha: G \rightarrow \operatorname{Diff}^{k}(M)$ we may define a bounded linear action of $G$ on $W^{s}(T M), d / 2<s<k$, called the adjoint action and denoted $\alpha_{\#}$. Here $W^{s}(T M)$ denotes the set of sections of $T M$ which are in $W^{s}(M, T M)$. (See the appendix for relevant definitions and theorems concerning the Sobolev spaces $W^{s}(M, N)$.) This adjoint action is given by

$$
\alpha_{\#}(g)(v)=D \alpha(g) \circ v \circ \alpha(g)^{-1} .
$$

Let $D R: W^{s}(T M)^{n} \rightarrow W^{s}(T M)^{m}$ denote the operator associated with $\alpha_{\#}$ as described in Chapter II, and let $H^{1}\left(G, W^{s}(T M)\right)$ be the first cohomology of $G$ with respect to the linear action $\alpha_{\#}$.

TheOREM 3.1. Let $\alpha$ be a $C^{k}$-action, where $d / 2<s \leqslant k-2$. If $H^{1}\left(G, W^{s}(T M)\right)=0$ and the image of $D R$ is closed then for all actions $\beta$ sufficiently close to $\alpha$ in $\operatorname{Act}^{k}(G, M)$ there is a map $h \in W^{s}(M, M)$ such that

$$
\beta(g) \circ h=h \circ \alpha(g)
$$

for all $g \in G$.

Proof. For any $C^{k}$-action $\beta$ in $\operatorname{Act}^{k}(G, M)$ define an action $[\beta, \alpha]$ on $W^{s}(M, M)$ by $[\beta, \alpha](g)(h)=\beta(g) h \alpha(g)^{-1}$. It is a result of Theorem A.2 (see the Appendix) that this is a well-defined $C^{k-s}$-action of $G$. By Theorem A.3, if $\beta$ is $C^{k}$-close to $\alpha$ then $[\beta, \alpha]$ is $C^{k-s}$-close to $[\alpha, \alpha]$. It is clear that $D[\alpha, \alpha](g)_{\text {id }}=\alpha_{\#}(g)$, for all $g$ in $G$, where id denotes the identity map of $M$. Also, by Theorem A.1, $W^{s}(T M)=$ $T_{\text {id }} W^{s}(M, M)$. Hence $[\alpha, \alpha]$ has id $\in W^{s}(M, M)$ as a stationary point and satisfies the hypothesis of Theorem 2.1. Therefore, if $\beta$ is sufficiently close to $\alpha$ in $\operatorname{Act}^{k}(G, M)$, there is a map $h \in W^{s}(M, M)$ such that $[\beta, \alpha](g)(h)=\beta(g) \circ h \circ \alpha(g)^{-1}=h$. The theorem follows.

The Sobolev Embedding Theorem implies that if $s=[d / 2]+1$, where $[d / 2]$ is the greatest integer less than or equal to $d / 2$, then $W^{s}(M, M) \subset C^{0}(M, M)$ and the inclusion is continuous. Hence, for $\beta$ sufficiently close to $\alpha$, the map $h$ in equation (5) may be taken to be homotopic to the identity and hence onto. For $s>[d / 2]+1$ the map is differentiable, and hence for $\beta$ sufficiently close to $\alpha$ we may take $h$ to be a diffeomorphism since $\operatorname{Diff}^{1}(M)$ is open in $C^{1}(M, M)$. We will say that an action $\alpha$ satisfying the conclusion of Theorem 3.1 for $s=[d / 2]+1$ is $S$-semistable. If, in addition, the map $h$ in (5) is a homeomorphism we say that $\alpha$ is $S$-structurally stable.

An application of Theorem 3.1. Let $S^{1}$ denote the circle and let $g$ be an orientation-preserving $C^{3}$-diffeomorphism of $S^{1}$ satisfying the following:

A. The set $F(g)$ of all fixed points of $g$ is finite and nonempty.

B. $D g(p) \neq 1$ for all $p \in F(g)$. 
It is classical that such diffeomorphisms are structurally stable. We will now see that $g$ is $S$-semistable.

Let $G$ be the (infinite cyclic) group generated by $g$.

Proposition 3.2. $H^{1}\left(G, W^{1}\left(T S^{1}\right)\right)=0$.

Proof. Let $p_{1}, \ldots, p_{n}$ be the fixed points of $g$. It is easy to see that $n$ is even and we may assume $D g\left(p_{i}\right)<1$ if $i$ is odd and $D g\left(p_{i}\right)>1$ if $i$ is even. Let $\left\{U_{i}\right\}_{i=1}^{2 n}$ be an open cover of $S^{\prime}$ satisfying the following conditions:

(a) $U_{i}$ is connected, $1 \leqslant i \leqslant 2 n$.

(b) $p_{i} \in U_{j}$ if and only if $j=2 i$.

(c) $D g(x) \neq 1$ if $x \in U_{2 i}, 1 \leqslant i \leqslant n$.

(d) If $i$ is odd then there is an integer $N>0$ such that $|j| \geqslant N$ implies that $g^{j}\left(U_{i}\right) \cap U_{i}=\varnothing$.

Let $\delta_{i}, 1 \leqslant i \leqslant 2 n$, be a smooth partition of unity subordinate to this cover. To prove the proposition we must show that $I-g_{\#}: W^{1}\left(T S^{1}\right) \rightarrow W^{1}\left(T S^{1}\right)$ is onto. This will be done by constructing a right inverse to $I-g_{\#}$ as in Robbin [14]. Let $v \in W^{1}\left(T S^{1}\right)$ and define $J_{i}(v), 1 \leqslant i \leqslant 2 n$, by

$$
J_{i}(v)=\sum_{j=0}^{\infty} g_{\#}^{j}\left(\delta_{i} v\right) \quad \text { if } i \neq 0(\bmod 4)
$$

and

$$
J_{i}(v)=-\sum_{j=1}^{\infty} g_{\#}^{-j}\left(\delta_{i} v\right) \quad \text { if } i \equiv 0(\bmod 4) .
$$

If $J_{i}, 1 \leqslant i \leqslant 2 n$, is well defined we have

$$
\left(I-g_{\#}\right) \sum_{i=1}^{2 n} J_{i}(v)=\sum_{i=1}^{2 n}\left(I-g_{\#}\right) J_{i}(v)=\sum_{i=1}^{2 n} \delta_{i} v=v \text {. }
$$

We proceed to show $J_{i}$ is well defined for each $1 \leqslant i \leqslant 2 n$.

Case 1. If $i$ is odd then the sum $\sum_{j=0}^{\infty} g_{\#}^{j}\left(\delta_{i} v\right)$ is finite by (d).

Case 2. If $i \equiv 2(\bmod 4)$ then $D g(x)<1$ for all $x \in U_{i}$. Let $p=p_{i}, U=U_{i}$ and $w=\delta_{i} v$.

We must show that $\sum_{j=0}^{\infty} g_{\#}^{j} w$ converges in $W^{1}\left(T S^{1}\right)$. We do this by showing that there is a constant $C$ and a positive real number $b<1$ such that $\left\|g_{\#}^{j} w\right\|_{1}^{2} \leqslant C b^{j}\|w\|_{1}^{2}$, where \|\|$_{1}$, is the norm in $W^{1}\left(T S^{1}\right)$.

A few lemmas are needed. Let

$$
\begin{aligned}
& a=\min \{|D g(x)| \mid x \in U\}, \\
& b=\max \{\mid D g(x) \| x \in U\}, \\
& c=\max \left\{\mid D^{2} g(x) \| x \in U\right\} .
\end{aligned}
$$

LEMMA 3.3. $D g^{j}(x) \leqslant b^{j}$ for all $j \geqslant 0$ and all $x \in U$.

Proof. This is immediate from the chain rule and the fact that $g(U) \subseteq U$.

LEMmA 3.4. $\left|D^{2} g^{j}(x) / D g^{j}(x)\right| \leqslant c /(a(1-b))$ for all $x \in U$ and $j \geqslant 1$. 
Proof. First, note that

$$
\frac{D^{2} g^{j}(x)}{D g^{j}(x)}=\sum_{i=0}^{j-1} \frac{D^{2} g\left(g^{i}(x)\right)}{D g\left(g^{i}(x)\right)} D g^{i}(x)
$$

is valid by induction on $j$. For $j=1,(6)$ is trivial and

$$
\begin{aligned}
\frac{D^{2} g^{j+1}(x)}{D g^{j+1}(x)} & =\frac{D^{2} g\left(g^{j}(x)\right) D g^{j}(x)^{2}+D g\left(g^{j}(x)\right) D^{2} g^{j}(x)}{D g\left(g^{j}(x)\right) D g^{j}(x)} \\
& =\frac{D^{2} g\left(g^{j}(x)\right)}{D g\left(g^{j}(x)\right)} D g^{j}(x)+\frac{D^{2} g^{j}(x)}{D g^{j}(x)}
\end{aligned}
$$

completes the induction step.

Using equation (6) we see that

$$
\begin{aligned}
\left|\frac{D^{2} g^{j}(x)}{D g^{j}(x)}\right| & =\left|\sum_{i=0}^{j-1} \frac{D^{2} g\left(g^{i}(x)\right)}{D g\left(g^{i}(x)\right)} D g^{i}(x)\right| \\
& \leqslant \sum_{i=0}^{j-1}\left|\frac{D^{2} g\left(g^{i}(x)\right)}{D g\left(g^{i}(x)\right)}\right|\left|D g^{i}(x)\right| \\
& \leqslant \sum_{i=0}^{j-1} \frac{c}{a} b^{i}=\frac{c}{a} \frac{1-b^{j}}{1-b} \leqslant \frac{c}{a(1-b)} .
\end{aligned}
$$

This completes the proof of the lemma.

The following calculation finishes the proof of the claim. Let $\int$ denote integration over $U$.

$$
\begin{aligned}
\left\|g_{\#}^{j} w\right\|_{1}^{2}= & \int\left(g_{\#}^{j} w(x)\right)^{2} d x+\int\left[D\left(g_{\#}^{j} w\right)(x)\right]^{2} d x \\
= & \int D g^{j}\left(g^{-j}(x)\right)^{2} w\left(g^{-j}(x)\right)^{2} d x \\
& +\int\left[D^{2} g^{j}\left(g^{-j}(x)\right) D g^{-j}(x) w\left(g^{-j}(x)\right)\right. \\
= & \left.\int_{g^{-j}(U)} D g^{j}(x)^{3} w(x)^{2} d x g^{j}\left(g^{-j}(x)\right) D w\left(g^{-j}(x)\right) D g^{-j}(x)\right]^{2} d x \\
& +\int_{g^{-j}(U)}\left[D^{2} g^{j}(x) D g^{-j}\left(g^{j}(x)\right) w(x)+D w(x)\right]^{2} D g^{j}(x) d x \\
= & \int D g^{j}(x)^{3} w(x)^{2} d x+\int\left[\frac{D^{2} g^{j}(x)}{D g^{j}(x)} w(x)+D w(x)\right]^{2} D g^{j}(x) d x
\end{aligned}
$$




$$
\begin{aligned}
\leqslant & b^{3 j} \int w(x)^{2} d x+2 \int\left[\frac{D^{2} g^{j}(x)}{D g^{j}(x)} w(x)\right]^{2} D g^{j}(x) d x \\
& +2 \int D w(x)^{2} D g^{j}(x) d x \\
\leqslant & \left(b^{j}+2 K^{2} b^{j}\right) \int w(x)^{2} d x+2 b^{j} \int D w(x)^{2} d x \quad \text { where } K=\frac{c}{a(1-b)} \\
\leqslant & C b^{j}\|w\|_{1}^{2} \quad \text { where } C=\max \left\{1+2 K^{2}, 2\right\} .
\end{aligned}
$$

This completes Case 2.

Case 3. If $i \equiv 0(\bmod 4)$ the argument which shows that $\sum_{j=1}^{\infty} g_{\#}^{-j} w$ converges is the same as the one given above for Case 2. This completes the proof of Proposition 3.2.

Since $D R$ is the zero map for $G$, we see that if $f$ is a $C^{3}$-diffeomorphism of $S^{1}$ which is sufficiently $C^{3}$-close to $g$ then there is an onto map $h \in W^{1}\left(S^{1}, S^{1}\right)$ such that $g \circ h=h \circ f$.

Proposition 3.5. Let $G=\left\langle a, b \mid a^{2}, b^{2}\right\rangle$ and let $\alpha: G \rightarrow \operatorname{Diff}^{3}\left(S^{1}\right)$ be a $C^{3}$-action such that $\alpha(a b)$ satisfies conditions $\mathrm{A}$ and $\mathrm{B}$. Then $H^{1}\left(G, W^{1}\left(T S^{1}\right)\right)=0$.

Proof. The proof uses the following fact (see [17]). Let $A$ be a $G$-Hilbert space and let $H$ be a subgroup of $G$ of finite index. Then $H^{1}(H, A)=0$ implies that $H^{1}(G, A)=0$. In Proposition 3.5 the subgroup generated by the element ab clearly has index two in $G$. The result then follows by Proposition 3.2.

Putting Propositions 2.4 and 3.2 together and applying Theorem 3.1 yields the following result.

Proposition 3.6. $\alpha$ is S-semistable.

Three theorems. Let $G$ and $H$ be finitely presented groups. Let $\alpha$ and $\beta$ be actions of $G$ and $H$ on some closed smooth manifold $M$, and let $\alpha * \beta$ be the action of $G * H$ on $M$ described in the last section of Chapter II.

THEOREM 3.7. Suppose $\alpha$ and $\beta$ satisfy the hypotheses of Theorem 3.1. Then $\alpha * \beta$ satisfies the hypotheses of Theorem 3.1 if and only if

$$
W^{s}(T M)=H^{0}\left(G, W^{s}(T M)\right)+H^{0}\left(H, W^{s}(T M)\right) .
$$

Proof. The Mayer-Vietoris sequence (see [17]) for $G * H$ is

$$
\begin{aligned}
0 & \rightarrow H^{0}(G * H) \rightarrow H^{0}(G) \oplus H^{0}(H) \stackrel{d}{\rightarrow} H^{0}(e) \\
& \rightarrow H^{1}(G * H) \rightarrow H^{1}(G) \oplus H^{1}(H) \rightarrow \cdots
\end{aligned}
$$

where the coefficients are understood to be $W^{s}(T M)$ and $e$ denotes the group with one element. Since $d(x, y)=x-y, H^{0}(e)=W^{s}(T M)$ and $H^{1}(G)=H^{1}(H)=0$ by hypothesis we see that $H^{1}(G * H)=0$ if and only if (7) holds. Proposition 2.4 now finishes the proof.

Let $\alpha$ be as above. We will say that $\alpha$ is expansive if there is some metric $d$ inducing the topology on $M$ and a positive real number $\varepsilon$ such that for every pair of distinct points $p$ and $q$ in $M$ there is an element $g \in G$ such that $d(\alpha(g) p, \alpha(g) q)>\varepsilon$. 
THEOREM 3.8. If $\alpha$ is expansive and satisfies the hypotheses of Theorem 3.1 then $\alpha$ is S-structurally stable.

Proof. We may choose $\beta$ sufficiently close to $\alpha$ so that $h$ in equation (6) satisfies $d(h(x), x) \leqslant \varepsilon / 2$ for all $x \in M$ and $h$ is onto. It suffices to show $h$ is one-to-one.

Let $p$ and $q$ be points in $M$ such that $h(p)=h(q)$. Then for all $g \in G$,

$$
h \alpha(g) p=\beta(g) h(p)=\beta(g) h(q)=h \alpha(g) q .
$$

Therefore,

$$
\begin{aligned}
d(\alpha(g) p, \alpha(g) q) \leqslant & d(\alpha(g) p, h \alpha(g) p)+d(h \alpha(g) p, h \alpha(g) q) \\
& +d(h \alpha(g) q, \alpha(g) q) \leqslant \varepsilon / 2+\varepsilon / 2=\varepsilon .
\end{aligned}
$$

But this contradicts the fact that $\alpha$ is expansive. Hence, $h$ is one-to-one.

Let $\alpha \in \operatorname{Act}^{k}(G, M)$ where $G$ is a finitely presented group of type $(F P)_{3}$ and $M$ is a smooth closed $d$-dimensional manifold. Let $s$ be an integer such that $d / 2<s<k$ -1 .

THEOREM 3.9. If $H^{1}\left(G, W^{s}(T M)\right)=0$ and $H^{2}\left(G, W^{s}(T M)\right)$ is finite dimensional then $\alpha$ is $S$-semistable. Furthermore, if $\alpha$ is also expansive then $\alpha$ is $S$-structurally stable.

Proof. This is a combination of Theorem 3.1, Proposition 2.2 and Theorem 3.8.

\section{APPENDIX}

Let $U$ be a bounded open subset of Euclidean space, $\mathbf{R}^{n}$, with smooth boundary. Let $\bar{U}$ be the closure of $U$. Define $C^{\infty}\left(U, \mathbf{R}^{m}\right)$ to be the set of functions from $U$ into $\mathbf{R}^{m}$ that can be extended to a $C^{\infty}$-function on some open set in $\mathbf{R}^{n}$ containing $\bar{U}$. To describe the Sobolev spaces $W^{s}\left(U, \mathbf{R}^{m}\right)$, we introduce norms ||$_{s}$ on $C^{\infty}\left(U, \mathbf{R}^{m}\right)$ as follows: Let $f \in C^{\infty}\left(U, \mathbf{R}^{m}\right)$ and recall that $D^{k} f: U \rightarrow L^{k}\left(\mathbf{R}^{n}, \mathbf{R}^{m}\right)$ where $L^{k}\left(\mathbf{R}^{n}, \mathbf{R}^{m}\right)$ denotes the set of $k$-linear maps from $\mathbf{R}^{k n}$ to $\mathbf{R}^{m}$ with the standard norm. Set

$$
|f|_{s}^{2}=\int_{U} \sum_{k=0}^{s}\left\|D^{k} f(x)\right\|^{2} d x .
$$

Now, $W^{s}\left(U, \mathbf{R}^{m}\right)$ is defined to be the completion of $C^{\infty}\left(U, \mathbf{R}^{m}\right)$ with respect to the norm ||$_{s}$. Note that $W^{s}\left(U, \mathbf{R}^{m}\right)$ is a Hilbert space with inner product,

$$
\langle f, g\rangle_{s}=\int_{U} \sum_{k=0}^{s}\left(D^{k} f(x) \cdot D^{k} g(x)\right) d x,
$$

where the inner product on $L^{k}\left(\mathbf{R}^{n}, \mathbf{R}^{m}\right)$ is induced from $\mathbf{R}^{m}$. The following theorem is useful in Chapter III.

Sobolev's Theorem. If $s>n / 2+k$ then $W^{s}\left(U, \mathbf{R}^{m}\right) \subseteq C^{k}\left(U, \mathbf{R}^{m}\right)$. When $C^{k}\left(U, \mathbf{R}^{m}\right)$ has the standard Whitney $C^{k}$-topology this inclusion is continuous.

Let $M$ and $N$ be smooth closed manifolds of dimension $m$ and $n$ respectively. Define $W^{s}(N, M)$ to be the space of functions $f: N \rightarrow M$ such that for any $p \in N$ 
and any chart $(U, \zeta)$ containing $p$ and any chart $(V, \psi)$ containing $f(p)$ the map $\psi \circ f \circ \zeta^{-1}: \zeta(U) \rightarrow \mathbf{R}^{m}$ is in the space $W^{s}\left(\zeta(U), \mathbf{R}^{m}\right)$. Using the Sobolev Theorem this definition can be shown to be independent of charts for $s>n / 2$. The case $s \leqslant n / 2$ is full of (possibly interesting) subtleties and will not be deal with here. In the following assume $s>n / 2$.

THEOREM A.1. $W^{s}(M, N)$ is a smooth Hilbert manifold whose tangent space at $f \in W^{s}(M, N)$ is

$$
T_{f} W^{s}(M, N)=\left\{v \in W^{s}(M, T N) \mid \pi_{N} \circ v=f\right\} .
$$

Let $f, g \in \operatorname{Diff}^{k}(M), k \geqslant s$, and let $[f, g]: W^{s}(M, M) \rightarrow W^{s}(M, M)$ by $[f, g](h)=$ $f \circ h \circ g^{-1}$.

TheOREM A.2. $[f, g]$ is a $C^{k-s}$-map of Hilbert manifolds.

THEOREM A.3. The assignment $f \rightarrow[f, g]$ for fixed $g$ is a continuous map,

$$
C^{k}(M, M) \rightarrow C^{k-s}\left(W^{s}(M, M), W^{s}(M, M)\right) .
$$

Notes. Sobolev's Theorem can be found in any book on Sobolev spaces, see for example [1]. The best references for Theorems A.1 and A.2 are the papers of Ebin, Fischer and Marsden [5, 6]. The proof of Theorem A.3 ị found in [9].

\section{REFERENCES}

1. R. A. Adams, Sobolev spaces, Academic Press, New York, 1975, p. 95.

2. R. Bieri, Homological dimension of discrete groups, Queen Mary College (University of London), London, 1976, pp. 19, 20.

3. C. Camacho, On $\mathbf{R}^{k} \times \mathbf{Z}^{\prime}$ actions, Dynamical Systems (M. M. Peixoto, ed.), Academic Press, New York, 1973, p. 23.

4. N. Dunford and J. T. Schwartz, Linear operators, Vol. 1, Interscience, New York, 1958, p. 487.

5. D. Ebin, A. Fischer and J. Marsden, Diffeomorphism groups, hydrodynamics and relativity, Proc. Thirteenth Bienniel Sem. Canad. Math. Congress, Vol. I (J. R. Vanstone, ed.), Canad. Math. Congress, Montreal, Canada, 1972, p. 135.

6. D. Ebin and J. Marsden, Groups of diffeomorphisms and the motion of an incompressible fluid, Ann. of Math. (2) 92 (1970), 102.

7. I. Kaplansky, Commutative rings, Allyn and Bacon, Boston, Mass., 1970, p. 137.

8. S. Lang, Introduction to differentiable manifolds, Interscience, New York, 1962.

9. S. Mizohata, Lectures on the Cauchy problem, Tata Institute of Fundamental Research, Bombay, 1965, p. 181.

10. R. S. Palais, Equivalence of nearby differentiable actions of a compact group, Bull. Amer. Math. Soc. 67 (1961), 362

11. J. Palis, Rigidity of centralizers of diffeomorphisms and structural stability of suspended foliations, Proc. Sympos. Pontifica Universidad Catolica do Rio de Janeiro (P. A. Schweitzer, S.J., ed.), Springer-Verlag, New York, 1978, p. 114.

12. C. Pugh and M. Shub, Ergodicity of Anosov actions, Invent. Math. 15 (1972), 1.

13. Axiom A actions, Invent. Math. 29 (1975). 7.

14. J. Robbin, A structural stability theorem, Ann. of Math. (2) 94 (1971), 447.

15. R. C. Robinson, Structural stability of $C^{1}$ flows, Proc. Sympos. (University of Warwick, 1973) (A. Manning, ed.), Springer-Verlag, New York, 1975, p. 262.

16. P. R. G. Sad, $A$ structurally stable $\mathbf{Z} \times \mathbf{Z}$ action, Proc. Amer. Math. Soc. 260 (1980), 515.

17. J.-P. Serre, Cohomologie des groupes discrets, Prospects in Mathematics, Princeton Univ. Press, Princeton, N. J., 1971, p. 90. 
18. S. Smale, Global stability questions in dynamical systems, Lectures in Modern Analysis, Vol. 1, Springer-Verlag, Berlin, 1969, p. 150.

19. __ Notes on differentiable dynamical systems, Proc. Sympos. Pure Math., Global Analysis, vol. 16, Amer. Math. Soc., Providence, R.I., 1970, p. 287.

20. D. Stowe, The stability of the stationary set of a group action, Proc. Amer. Math. Soc. 79 (1980), 139.

21. A. Weil, Remarks on the cohomology of groups, Ann. of Math. (2) 80 (1964), 149.

Department of Mathematics and Statistics, Case Western Reserve University, Cleveland, ОНIO 44106

Current address: Bell Laboratories, Short Hills, New Jersey 07078 